\title{
'What I said' versus 'What you heard': a comparison of physicians' and parents' reporting of anticipatory guidance on child safety issues
}

\author{
Barbara A Morrongiello, Loretta Hillier, Martin Bass
}

\begin{abstract}
Objective-Unintentional injuries are the number one cause of death for infants. Many of these injuries could be prevented if parents took additional safety precautions. In this study physicians' and parents' perspectives regarding the part that physicians play in educating first time parents about child safety issues were compared.
\end{abstract}

Methods-All pediatricians and family physicians in London, Ontario were surveyed by mail (68\% return rate) regarding their practices, attitudes, and beliefs related to parent education about child safety issues. A sample of 114 first time mothers, including 38 each with 6,12 , and 18 month old infants, completed a telephone interview. All parents had physicians who had returned questionnaires.

Results-There was good correspondence between parents' and physicians' judgments about the safety issues most often covered, and what role physicians should adopt regarding parent education about child safety issues. In addition, they both agreed that parents seldom seek out safety information by asking questions. Relative to parent reports, however, physicians significantly overestimated the time they spent on safety issues and the degree of their direct involvement in communicating this information. The best predictor of time spent by physicians on safety issues was their rating of the importance of assuming the role of parent educator. The best predictor of parents asking questions about child safety was their rating of the adequacy of physicians' responses to previously asked questions.

Psychology

Department,

University of Guelph,

Guelph, Ontario N1G

2W1, Canada

B Morrongiello

University of Western Ontario, London,

Canada

L Hillier

M Bass

Correspondence and reprint requests to: $\mathrm{Dr}$ Morrongiello.
Although childhood deaths due to injuries have declined over the past 20 years, in most industrialized countries, unintentional injuries still both physicians and parents contribute to undermine communication about child (Injury Prevention 1995; 1: 223-227) Keywords: physician-parent communication, physician
counseling. rank as the leading cause of death for infants and children who were otherwise healthy. ${ }^{12}$ In fact, in North America injuries account for more loss of life in 1 to 14 year olds than the next six leading causes of death combined. ${ }^{34}$ One out of every three pediatric deaths is due to injury. ${ }^{5}$ Unfortunately, in spite of years of research, we still have a relatively limited understanding of the best ways to prevent childhood injuries. Prevention of injuries therefore remains a topic of considerable interest in the medical and psychological literatures. ${ }^{67}$

Many intervention programs have been aimed at injury prevention in children. The public health approach focuses on environmental interventions that alter the hazardous situation, such as packaging medicine in childproof containers or installing window guards in apartment buildings occupied by children. ${ }^{89}$ These approaches are usually successful and benefit the lives of large numbers of children. However, not many safety hazards can be dealt with by such passive recipient approaches. Community education or counseling interventions often involve mass media programs, and often yield only limited success. ${ }^{10}$ Behavioral based intervention programs frequently provide rewards and modeling to increase safety promotion behaviors. ${ }^{11}$ These programs yield positive effects, but are costly to implement and the new behaviors are not always maintained after the program ends. ${ }^{12}$

An alternative approach to injury prevention is to make use of resources already in place and with which patients have frequent contact, specifically, physicians. ${ }^{13}$ Micik and Alpert argue persuasively that physicians are in the best position to educate parents about safety issues because they appreciate how injury risk varies with developmental level; they have status as respected experts on child development that should make parents more likely to consider their advice; and they can repeatedly reinforce parents' knowledge and injury prevention practices. ${ }^{14}$ Consistent with these points, a study by Bass and colleagues clearly illustrates the beneficial effect counseling about safety by physicians can have on parents' behaviors. ${ }^{15}$ When compared with a control group that did not receive such counseling, the homes of the counseled parents had significantly fewer 'at risk' items. In fact, a recent review reveals that in 18 out of 20 studies, there were positive effects of injury 
prevention counseling to parents by health care professionals, in most cases, physicians. ${ }^{16}$

Recognizing the potential for physicians to significantly influence parents' safety practices, anticipatory guidance on safety issues is endorsed by the American Academy of Pediatrics and the Canadian Medical Association. Although physicians are apparently aware of the need for preventive counseling about child safety, ${ }^{15}$ there is some question in the literature as to how much time physicians actually spend doing so. For example, Reisinger and Bires found that physicians spent an average of 87 seconds per well-baby session counseling parents of 6 to 11 month olds, of which only 12.4 seconds were devoted to injury prevention! ${ }^{17}$

The aim of this study was to compare physicians' self reports with parent reports regarding the time physicians spend on child safety issues, the topics covered, and how the information was delivered. In addition to actual reported practices, we also compared attitudes about the role of physicians as parent educators, perceived barriers that prevented physicians spending more time on safety issues, and beliefs about parents' sources of safety knowledge. Finally, we compared physicians' and parents' perspectives on the part parents play in seeking safety information by asking questions.

\section{Subjects and methods}

SUBJECTS

Using the Canadian Medical Association directory and the local telephone directory, al pediatricians $(\mathrm{n}=12)$ and family physicians $(n=197)$ in London, Ontario were mailed questionnaires to assess their practices, attitudes, and beliefs about the role of physicians in educating first time parents on child safety issues. London has a population of approximately 300000 and includes three major hospitals and a medical school. Of the physicians who returned the questionnaires, $60 \%$ had been in practice for 10 or more years.

The names of 114 first time mothers were obtained from a database of families with children born at St Joseph's Health Centre in London. These parents were listed on the database because they had indicated an interest in research participation at the time the child was born. To maximize variability in social class and ethnic group, and ensure a broad sampling of patients of respondent physicians, we divided the city into four quadrants on a map and selected the parent sample to be equally distributed across these quadrants. The physician sample was similarly distributed. All primary caretaker participants were mothers, all of whom had physicians who had returned a questionnaire. Since safety issues vary with infant age, and injury statistics vary with child's sex, the sample included mothers of 38 babies in each of three age groups: 6,12 , and 18 months, with an equal number of boys and girls in each age group. An additional 23 parents were contacted but excluded because their physician had not completed the questionnaire.
MATERIALS AND METHODS

The physician questionnaire included 18 items that assessed their practices, attitudes, and beliefs related to educating first time parents on child safety issues. A copy of the questionnaire and data coding instructions are available from the first author upon request. With regards to their actual practices, we asked how often they provide information about safety issues, how this information is provided, what issues are covered, and how often parents request information or advice on these issues. With regards to attitudes and beliefs, we took their ratings of the importance of physicians in providing safety information to parents: how reasonable it was to expect physicians to adopt the role of educator, their beliefs about the three most common sources that parents use for safety information, the perceived barriers to physicians spending more time on safety issues, and whether their medical training adequately prepared them to educate parents on safety issues.

The parent telephone interview began by asking parents about whether they took their child for well-baby visits. Subsequently, they were asked to describe the last injury of any type the infant experienced and to rate the extent to which it could have been prevented. Parents were told to consider minor mishaps that made the infant cry and resulted in tissue damage, as well as more significant mishaps that required medical intervention. They were also asked to indicate whether the child had, or had almost, experienced any of a number of other injuries. Parents were then asked whether they had discussed a number of safety issues with their physician at any time, and to indicate who had initiated the discussion. Parents also rated how often the physicians provided information about safety, how this information was provided (verbal, pamphlet, poster), and what issues were covered.

To assess the communication exchange between parents and physicians, we also asked parents to rate how often they requested information or advice on safety/injury prevention from their physician, how comfortable they felt doing so, how receptive their physician was to such discussions, and the adequacy of their physician's previous responses when they sought advice.

With regards to attitudes and beliefs, we assessed their ratings of the importance of physicians providing safety information to parents, how reasonable it was to expect physicians to adopt such an educator role, the most common source they use for safety information, and the barriers they perceived to physicians spending more time on safety issues.

\section{PROCEDURE}

Questionnaires were mailed to physicians, along with an explanatory letter and a return postcard. Two follow up telephone calls were made to physicians who did not respond. The calls occurred at two week intervals, and additional copies were mailed if requested. This modified Dillman method ${ }^{18}$ resulted in a return rate of $68 \%$. 
The postcard, which had a physician identification code on it, was returned under separate cover from the questionnaire, thereby allowing us to compile a list of physicians who had returned the questionnaire while maintaining their anonymity.

To gather the data from parents, the interviewer scheduled a 15 minute period. The interviewer was not aware of the physician data, and followed a structured interview format. At the conclusion of the interview, parents confirmed that their physician was on our list of participants. To do so, parents gave us the first initial of the last name of the physician, were read the list of corresponding physicians, and then indicated whether or not their physician's name was read. They were not asked to name their physician directly. All procedures were approved by the ethics review board at the university.

\section{Results}

There were no differences between family physicians and pediatricians in any comparisons to be reported, so we do not discuss results separately for these groups. The physicians estimated that $95 \%$ of parents in their practice brought their infants for wellbaby visits. This figure corresponded well to, and was not statistically different from, the $98 \%$ of parents in the sample who reported doing so. Physicians in our sample reported providing primary care for on average 18 infants a week (standard deviation:14 infants). Considering both well-baby and illness related visits, $61 \%$ of parents reported having taken their infant to the doctor eight to 10 times, and $38 \%$ indicated doing so at least four to seven times since the baby's birth. Thus, physicians had ample opportunity to provide anticipatory guidance on child safety and the parents had sufficient opportunity to be exposed to their physician doing so.

There was significant disagreement between the physicians' and parents' estimates of how often physicians spent time on safety issues during well-baby visits. About $55 \%$ of physicians reported that they mentioned age appropriate safety issues 'always' or 'most of the time', whereas only $19 \%$ of the parents agreed that their physicians did so 'always' or 'most of the time' $\left(\chi^{2}=33.15, \mathrm{p}<0.01\right)$. In fact, $64 \%$ of parents reported that their physician 'rarely' or 'never' discussed child safety issues spontaneously.

Similarly, when asked to indicate how safety information was conveyed, there was significant disagreement: $34 \%$ of parents indicated their physicians discussed the safety issue themselves with the parent, whereas $91 \%$ of physicians reported they they themselves communicated the safety information to parents $\left(\chi^{2}=85 \cdot 18\right.$, $\mathrm{p}<0.01)$. There was no disagreement, however, regarding the role ascribed to the nurse: $19 \%$ of physicians indicated the nurse provides safety information to parents and $20 \%$ of parents endorsed this statement. Similarly, there was virtually no disagreement regarding the use of posters and/or pamphlets to convey safety information to parents (approximately $32 \%$ of parents and physicians agreed with this). Thus, the disagreement between physician and parent ratings was specific to the part played by the physician directly.

Of the 26 safety issues covered, only two were reported with any regularity by parents and physicians alike as being covered in some way by physicians - namely, hazards of walkers and the use of car seats. Parents and physicians were in nearly perfect agreement on their ranking of these two topics as the most often covered. However, only $27 \%$ and $29 \%$ of parents reported that car seats and walkers, respectively, were mentioned by their physicians. Some of the topics listed might be construed as being unreasonable to spend time on (for example, safety features of highchairs or strollers, Ipecac Syrup, a first aid kit at home, or the need for constant supervision in play, etc). There were a number of topics relevant to preventing significant injuries in infants, however, that parents $(98 \%)$ reported as 'never' having been addressed by physicians. These included: use of gates inside the home $(85 \%$ said 'never'), window and screen locks (94\%), tap water/water heater temperature $(86 \%)$, microwave heating of infant food $(87 \%)$, and electric outlet covers $(89 \%)$.

Regarding their judgment of how important it was for physicians to assume an educational role in promoting safety, $67 \%$ of physicians indicated it was 'extremely' or 'very' important to do so, and $70 \%$ of parents gave similar ratings. However, only $48 \%$ of parents and $49 \%$ of physicians felt that it was 'extremely' or 'very' reasonable to expect physicians to fulfil this role, given the realities of clinical practice. These ratings indicate that parents and physicians both have similar values about the role physicians should have in safety promotion, however, they are similarly sensitive to the pragmatics facing primary care providers. Consistent with this interpretation, the barrier most often mentioned by parents and physicians alike, was the limited time physicians had for each patient.

Physicians and parents also differed in their ranking of the most common sources parents use for safety information. For parents, the most common sources reported were baby books and other parents $(71 \%$ endorsed these as their primary sources). By contrast, physicians indicated that 'common sense' was the most common primary source $(68 \%$ endorsed this). Consulting with other parents was ranked second by physicians $(49 \%)$. The belief that child safety is largely a matter of common sense may explain why a greater number of physicians do not spend more time educating parents about child safety issues, as reported by parents. In essence, why spend precious time on issues that parents could reach conclusions about for themselves?

Consistent with this interpretation, several physicians wrote that they did not realize the importance of anticipatory guidance about child safety until they had their own children. In fact, a stepwise regression analysis confirmed that the best predictor of physicians' 
time spent on child safety was their ratings of the importance of assuming the role of parent educator. This factor accounted for $29 \%$ of the variance. Another significant, but less influential, factor was use of a well-baby checklist ( $6 \%$ of variance).

Regarding whether parents themselves seek out safety information from the physician, only $4 \%$ indicated they 'always' ask for such information or advice, and $64 \%$ said they 'never' or 'rarely' had done so. Consistent with these parent ratings, $61 \%$ of physicians rated that parents 'never' or 'rarely' asked questions on safety matters. This lack of initiative did not seem to be due to problems of relating to their physicians because $82 \%$ believed the physician would be 'extremely' receptive to questions, and $92 \%$ reported that they felt 'extremely' comfortable interacting with him or her. Rather, the barrier seems limited to the content of physicians' previous replies. In this case, the best predictor of asking questions was the parents' rating of the adequacy of the physician's reply the last time they did so. This accounted for $26 \%$ of the variance in a regression aimed at predicting parent questioning of physicians about safety matters.

The injury rate for infants in this sample was $75 \%$ (most were categorized as falls, cuts, crushing injuries, or burns), and $60 \%$ of parents rated that these injuries could have been completely prevented by taking extra precautions or providing better supervision. With this in mind, it is important to note that $82 \%$ indicated that if physicians spent more time on safety education they believed they would be especially likely to follow the physician's advice and make changes to their own behaviors. Obviously, parents hold the opinion of their physician in high regard and feel they would be willing to act differently if he or she advised them to do so in order to better ensure their child's safety.

\section{Discussion}

Relative to parents' perceptions, physicians overestimated the time they themselves spent on safety counseling and the extent of their direct involvement. While one might hypothesize that somehow we sampled parents who were biased toward negative reporting, and they therefore underestimated the actual time and involvement of their physician, this seems unlikely. In fact, parents were sympathetic to the time constraints facing physicians and acknowledged that the realities of clinical practice made it unreasonable to expect extensive coverage of child safety. Thus, they did not seem unduly negative. Furthermore, parents' ratings of other aspects of the physicians' practice (for example, use of safety posters or pamphlets) was exactly in accord with ratings by physicians. This suggests that if parent reporting was negatively biased it would have to be specific to physicians' behaviors. There is no reason to think this is the case. None the less, because we did not observe physicians directly, it cannot be established with certainty which respondent is the more accurate reporter. We can say, however, that parents are obviously not viewing their physician as a primary source of safety information. This is unfortunate because parents have repeated contacts with their physicians during their child's first few years and could certainly benefit from viewing the physician as a resource for this information.

Physicians and parents apparently agree on the importance of physicians assuming an educator's role. However, our findings suggest that neither assumes an adequate share of responsibility to make this happen. From the parent's perspective, physicians do not spend much time on safety issues; at least, parents do not remember their physicians doing so. Conversely, parents do not seek such information from the physician. Thus, both parties contribute to the problems of limited communication about child safety.

There may be several barriers to communication operating here. First, a physician's belief that most child safety is 'common sense' may limit enthusiasm for explicit discussion of injury prevention. Training physicians to counsel more on safety might best proceed by focusing on their beliefs about injury prevention, as opposed to providing them injury statistics or prevention strategies. In fact, $63 \%$ of physicians in this sample judged that medical school had not adequately prepared them for safety counseling. Although there has been an increase in training focused on behavioral and developmental pediatrics since 1978, and physicians apparently feel positively about this training, ${ }^{19}$ more emphasis on anticipatory guidance related specifically to safety may be needed.

Another barrier to communication comes from the finding that parents draw on past exchanges with their physician in deciding whether or not to pursue a topic by questioning the physician. The fact that adequacy of physicians' previous responses to such questions best predicted the likelihood of future question asking suggests that physicians need to pay careful attention to the nature and scope of responses they provide. Failure to do so may impede the communication cycle, compromise the patient's use of physicians as a resource in the future, and ultimately put their health, or that of their infant, at risk.

Finally, it is worth pointing out the obvious: time spent on injury prevention will likely result in less time spent addressing injury consequences. Although limited time was acknowledged as a primary barrier by physicians and parents alike, better planning of resource allocations could substitute for direct access to the physician for safety education. Most issues could be narrowed by assuming a developmental perspective, and limiting oneself to the few items each visit that are most pertinent to the child's developmental status. This is the approach outlined in TIPP (The Injury Prevention Program), which is advocated by the American Academy of Pediatrics. Such an approach has been proven to maximize effective use of physicians' time. ${ }^{15}$ Similarly, group well-child care, an alternative to one-on- 
one well-baby visits, is another effective way to balance time pressures with the need to educate parents about injury prevention. ${ }^{2021}$

The injury rate in our sample was $75 \%$, with $60 \%$ of these events rated by the parents as preventable. Assuming that injury prevention is largely 'common sense' is obviously not keeping infants and children safe. Physicians need to take more time, and parents need to assume more responsibility and ask more questions, to promote greater discussion of child safety and injury prevention during well-baby visits.

This research was supported by a grant to the first author from the Hospital for Sick Children Foundation. The authors acknowledge the assistance of Krista Thompson in data collection.

1 Rodriguez J. Childhood injuries in the United States: a priority issue. Am F Dis Child 1990; 144: 625-6.

2 Rivara FP, Alexander B, Johnston B, Soderberg $R$. Population-based study of fall injuries in children and adolescents resulting in hospitalization or death. Pediatrics 1993; 92: 61-3.

3 Dershowitz R, Williamson J. Prevention of childhood household injuries: a controlled clinical trial. Am $\mathfrak{f}$ Public Health 1977; 67: 1148-53.

4 Canadian Institute of Child Health. The health of Canada's children. 2nd Ed. Ottawa: Canadian Institute of Child Health, 1994

5 Task Force on Pediatric Education. The future of pediatric education. Evanston, Illinois: American Academy of Pediatrics, 1978.
6 Finney J, Christophersen E, Friman P, et al. Society of Pediatric Psychology Task Force: pediatric psychology and injury control. F Pediatr Psychol 1993; 18: 499-526.

7 Roberts M. Special section editorial: explicating the circumstances of nonintentional injuries in childhood. $f$ Pediatr Psychol 1993; 18: 99-103.

8 Spiegel C, Lindaman F. Children can't fly: a program to prevent child mortality and morbidity from window falls. Am f Public Health 1977; 67: 1143-7.

9 Wolton W. An evaluation of the Poison Prevention Packaging Act. Pediatrics 1982; 69: 363-70.

10 Colver A, Hutchinson P, Judson E. Promoting children's home safety. $B M F$ 1982; 285: $1177-80$.

11 Roberts M, Turner D. Rewarding parents for their children's use of safety seats. $\mathcal{F}$ Pediatr Psychol 1986; 11: ren's us

12 Peterson L, Mori L, Rosen B. Interventions in children's injury prevention: differing costs and differing benefits. fournal of Community Psychology 1988; 16: 188-203.

13 Lovejoy L, Chafee-Bahanon C. The physician's role in accident prevention. Pediatr Rev 1982; 4: 53-60.

14 Micik S, Alpert J. The pediatrician as advocate. Pediatr Clin North $A m$ 1985; 31: 243-9.

15 Bass J, Mehta K, Ostrovosky M, Halperin S. Educating parents about injury prevention. Pediatr Clin North Am 1985; 32: 233-42.

16 Bass J, Christoffel K, Widome M, et al. Childhood injury prevention counseling in primary care setting: a critical review of the literature. Pediatrics 1993; 92: $544-50$.

17 Reisinger $\mathrm{K}$, Bires J. Anticipatory guidance in pediatric practice. Pediatrics 1980; 66: 889-92.

18 Dillman $\mathrm{D}$. The design and administration of mail surveys. Annual Review of Sociology 1991; 17: 225-49.

19 Breunlin D, Mann B, Richtsmeier A, et al. Pediatricians' perceptions of their behavioral and developmental training. Developmental and Behavioral Pediatrics 1990; 11 : $165-9$

20 Osborn L. Group well-child care. Pediatr Clin North Am 1985; 12: 355-66.

21 Thomas K, Hassaneim R, Christophersen E. Evaluation of group well-child care for improving burn prevention practices in the home. Pediatrics $1984 ; 74: 879-82$.

\section{Observed without comment}

Safe Community News, reporting on a seminar in India, included the following intriguing quotations: 'All the vehicles on the road are not in good condition, and brake failure is common because of poor maintenance and corrupt certification'. Another, describing bus crews, states, 'Most do not drink and drive, but $5 \%$ are forced to drink because of a very heavy and tight time schedule which gives no proper rest'. (A Ramalingam). (Editor: the italics are mine.)

\section{Most interesting paper title?}

In the last issue I initiated a contest for the most interesting (or amusing) title. My colleague, Nick Rodrigo, brought the following to my attention: 'Spontaneous pneumothorax, pneumomediastinum, and pneumopericardium in a 16 year old drug-abusing motorcyclist surrounded by a pack of coyotes' (Postgraduate Medicine 1989; 86: 79-80). This will be hard to beat, but do try! 